\title{
等离子激元纳米颗粒组装及其生物应用
}

\author{
张磊，莫兰，沈晶晶，张颖，汪联辉"
}

南京邮电大学信息材料与纳米技术研究院, 有机电子与信息显示国家重点实验室培育基地, 先进生物与化学制造协同创新中心, 南京 210023

* 联系人, E-mail: iamlhwang@njupt.edu.cn

2016-06-22 收稿, 2016-08-07 修回, 2016-08-10 接受, 2016-09-08 网络版发表

国家重点基础研究发展计划(2012CB933301)、国家自然科学基金(61302027, 21305070, 21475064, 61571239)、教育部长江学者和创新团队 发展计划(IRT_15R37)、教育部高校博士点专项基金(20123223120011, 20123223110007)、江苏高校优势学科建设工程(PAPD)和江苏省自然 科学基金(BM2012010, NY211003)资助

摘要近年来, 通过自组装技术能够可控地将单个纳米颗粒组装成一维、二维或三维空间构象的新型复合材料. 相对于单独的纳米颗粒, 纳米颗粒组装材料可表现出许多新颖或优异的物理性质, 具有巨大的应用前景, 因此在 纳米材料领域越来越受到关注并快速发展. 尤其是贵金属纳米颗粒组装体具有独特的等离子激元光学性质，在生 物传感、成像和治疗等方面具有广阔的应用前景. 本文回顾了近年来通过分子介导方法对纳米颗粒进行组装及其 生物应用的最新研究进展, 重点介绍了几种分子介导的纳米颗粒组装技术, 以及纳米颗粒组装体的生物医学应用, 并展望了其发展前景。

关键词纳米颗粒, 自组装, 分子介导, 生物应用

1957年, Ritchie ${ }^{[1]}$ 发现, 高能电子束在穿透金属 介质时, 能够激发出金属中自由电子(d电子)在正离 子背景中的量子化振荡运动, 产生等离子共振效应. 通常情况下, 金属和半导体中存在的大量自由电子 可以自由运动, 当其与特定波长的人射光波相互作 用时, 在电磁干扰作用下其电子密度的起伏将会引 起整个电子系统的集体运动, 形成等离子体振荡, 即 产生表面等离子共振散射现象. 由于该现象主要发 生在表面, 因此被称为表面等离子共振 (surface plasmon resonance, SPR)散射. 根据Mie理论, 等离子 激元 (plasmon)的散射光谱主要依赖于纳米颗粒(如 $\mathrm{Au}$ 膜、Au纳米颗粒、 $\mathrm{Ag}$ 纳米立方体等)的形状、大小、 距离以及颗粒本身及其周围环境的介电常数. 基于 单颗粒SPR效应, 其散射光谱可灵敏反应出等离子激 元界面的介电常数的细微变化.

与单独的纳米颗粒相比, 等离子激元纳米颗粒
组装体不仅具有纳米颗粒的特性，如量子尺寸效应、 小尺寸效应、表面效应和宏观量子隧道效应, 又具有 由纳米颗粒组合引起的新现象. 例如，当两个金纳米 颗粒(AuNPs)间距足够小时, AuNPs间的表面等离子 共振波将发生耦合效应，导致颗粒间隙处的电磁场 得到显著增强，在拉曼增强、金属催化和传感领域具 有广泛的应用 ${ }^{[2 \sim 5]}$. 此外, 通过改变组装纳米颗粒的 形状和大小 ${ }^{[6]}$, 调节组装颗粒间的距离 ${ }^{[7]}$, 或控制界 面分子取向和组装体维度 ${ }^{[8]}$ 等还可以实现对组装体 物理、光学性能的进一步调控. 近年来, 纳米颗粒自 组装技术取得了长足的发展并越来越受到关注. 纳 米颗粒自组装是指通过先制备低维纳米材料, 然后 再将其以“自下而上(bottom-up)”的方式进行后续自 组装获得各种超结构. 自组装过程并不是大量原子、 离子、分子之间的简单叠加, 而是若干个体之间通过 分子间特殊的相互作用, 如静电吸引、氢键、疏水性 
作用、生物分子识别作用等组装成有序的纳米结构, 是一种整体的复杂协同作用.

目前, 已报道的等离子激元纳米颗粒组装体形 态主要包括二聚体、三聚体、Janus纳米颗粒、线性 纳米链、网络阵列、球形簇和核心-卫星构造等. 这 些结构主要基于先在贵金属纳米颗粒界面联接功能 分子使其表面功能化, 之后再利用化学方法、静电作 用或直接通过功能分子的特异结合作用诱导其自组 装. 直接利用功能分子介导纳米颗粒组装成具有固 定结构的组装体是目前研究最多的组装方式, 为了 实现定向、可控组装, 已报道的纳米颗粒组装技术采 用了多种介导分子, 主要包括DNA、抗原-抗体特异 性反应、生物素-亲和素系统、有机小分子和线性、树 枝状、超支化聚合物等. 分子介导的等离子激元纳米 颗粒组装在生物传感、成像和医学治疗等方面具有广 泛的应用 ${ }^{[9 \sim 11]}$.

本文主要聚焦于分子介导的等离子激元纳米颗 粒组装及其生物应用, 回顾了近几年来几种分子介 导的纳米颗粒组装进展, 综述了分子介导的纳米颗 粒组装在比色法传感、表面增强拉曼光谱(SERS)传 感、SPR传感、手性传感及光热治疗与生物成像等生 物应用方面的最新研究成果, 分析了纳米颗粒自组 装及其在生物应用中仍待突破的一些难点, 并展望 了其发展前景.

\section{1 分子介导的纳米颗粒组装}

分子介导的纳米颗粒组装技术, 主要是利用生 物分子的特异性结合作用, 如DNA杂交、抗原-抗体 特异性反应、生物素-亲和素系统以及有机小分子和 聚合物等, 实现纳米颗粒定向自组装并形成特定的 结构.

\subsection{DNA}

具有互补碱基序列的DNA分子，可以利用碱基 对间形成氢键, 形成稳定的双链结构, 这种碱基配对 原则具有高度特异性. 除此之外, 由于DNA序列具 有可编程性和易操作等特点, 可被广泛地应用于构 建有序的纳米组装体, 形成一维、二维、三维不同构 型 ${ }^{[12]}$.

修饰 AuNPs的一个经典方法是利用强的 $\mathrm{Au}-\mathrm{S}$ 共 价键将颈基化的单链寡核苷酸(ssDNA)联接到AuNPs 上, 负载颈基ssDNA链的AuNPs通常表现出很强的杂
交能力 ${ }^{[13]}$. 1996年, Alivisatos等人 ${ }^{[14]}$ 首先采用DNA联 接 2 个独立的AuNPs, 颗粒间的距离可以通过杂交后 形成的刚性双链DNA(dsDNA) 的长度进行调控. 该 原理也被Mirkin课题组 ${ }^{[15]}$ 进一步用来组装纳米颗粒 超晶格结构, 如面心立方体 (FCC) 和体心立方体 (BCC) 金纳米超晶格(图 1(a)). 银纳米颗粒和纳米棒 也可以用硫基ssDNA功能化后组装形成二聚体、四聚 体以及超晶格结构, 并且与金纳米组装体相比, 银纳 米组装体呈现出更强烈的等离子共振效应 ${ }^{[17,18]}$.

近来, Fan研究组 ${ }^{[19]}$ 发现 ssDNA序列中腺嘌呤 (poly A)对AuNPs具有独特的作用力, 可吸附在AuNPs 表面，用仅含有poly A的ssDNA可同样实现AuNPs功 能化. 后来, 他们进一步调控含有poly A 的寡核苷酸 的长度来修饰 AuNPs, 获得了高产率单活性位点的 DNA-AuNP复合物，该方法使纳米颗粒组装更为简 单方便，条件更为温合，类似于有机合成中的“点击 化学”方法. 这些单活性位点的DNA-AuNP复合物可 为组装金等离子纳米结构提供精确的交联剂 ${ }^{[20]}$.

与单链DNA相比, DNA折纸具有更强的刚性和 更好的可编程性，因此可以通过灵活地设计DNA折 纸来组装复杂的等离子纳米结构. Liedl研究组 ${ }^{[16]}$ 利 用刚性DNA折纸支架作为交联剂将金属纳米颗粒、量 子点和有机染料组装成“卫星”结构的多层三维纳米 簇 (图 1(b)), 并且通过不同尺寸的DNA折纸作为交联 剂，可调控纳米簇中颗粒间的距离为 5 200 $\mathrm{nm}$. 他 们还指出, 可以通过化学沉积金或银使这些纳米团 簇的尺寸进一步扩大，该成果在等离子激元研究领 域具有巨大的应用前景.

适配体是一小段经体外篮选得到的寡核苷酸序 列，能与相应的配体进行强特异性和高亲和力的结 合. 蛋白质、氨基酸、药物、金属离子等, 甚至病毒、 完整的细胞、孢子等都可以作为适配体的靶分子. 适 配体容易修饰，与靶分子识别能力强，结合特异性 高. 因此，通过适配体的特异性识别诱导等离子激元 纳米颗粒组装逐渐成为研究热点. Xu研究组 ${ }^{[21]}$ 用能 与前列腺特异抗原(PSA)靶结合的适配体和与其部分 互补的DNA来组装卫星纳米结构. 没有PSA时, 适配 体修饰的核心金银核壳纳米颗粒与互补DNA联接的 卫星AuNPs相互结合形成卫星结构; 加人PSA后, 适 配体与PSA靶结合, 卫星纳米颗粒与核心纳米颗粒 分离(图2). PSA的SERS信号强度与核心纳米颗粒周 围的卫星纳米颗粒数量直接相关, 因此, PSA的浓度 

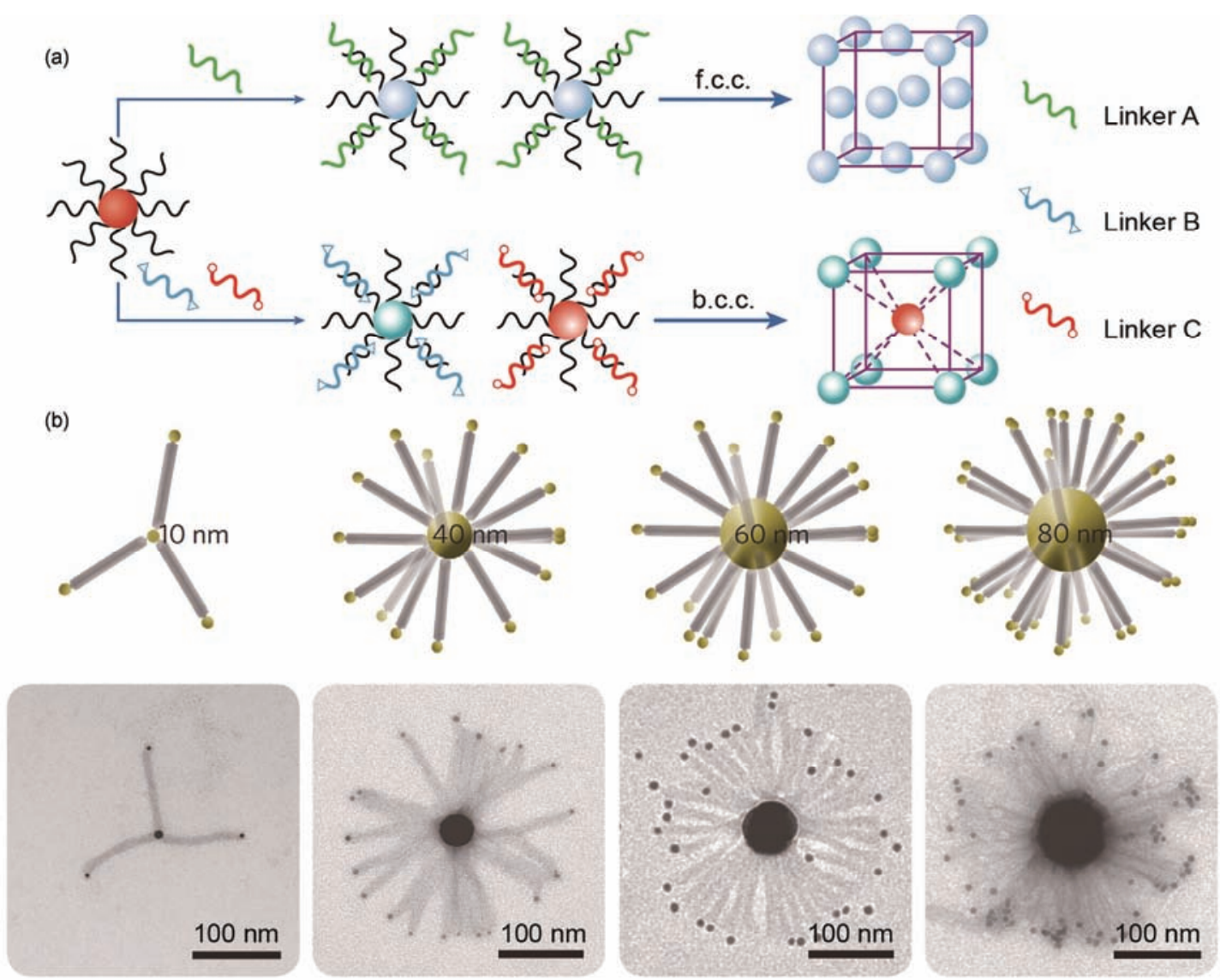

图 1 (网络版彩色)DNA介导的纳米颗粒组装. (a) 用不同序列的DNA链将AuNPs组装成不同超晶格 ${ }^{[15]}$; (b) 用DNA折纸支架分层组装纳米颗粒 ${ }^{[16]}$

Figure 1 (Color online) Scheme of DNA-mediated nanoparticles assemblies. (a) DNA-mediated assemble to different superlattice structure ${ }^{[15]}$. (b) Planet-satellite stoichiometry control by DNA origami ${ }^{[16]}$

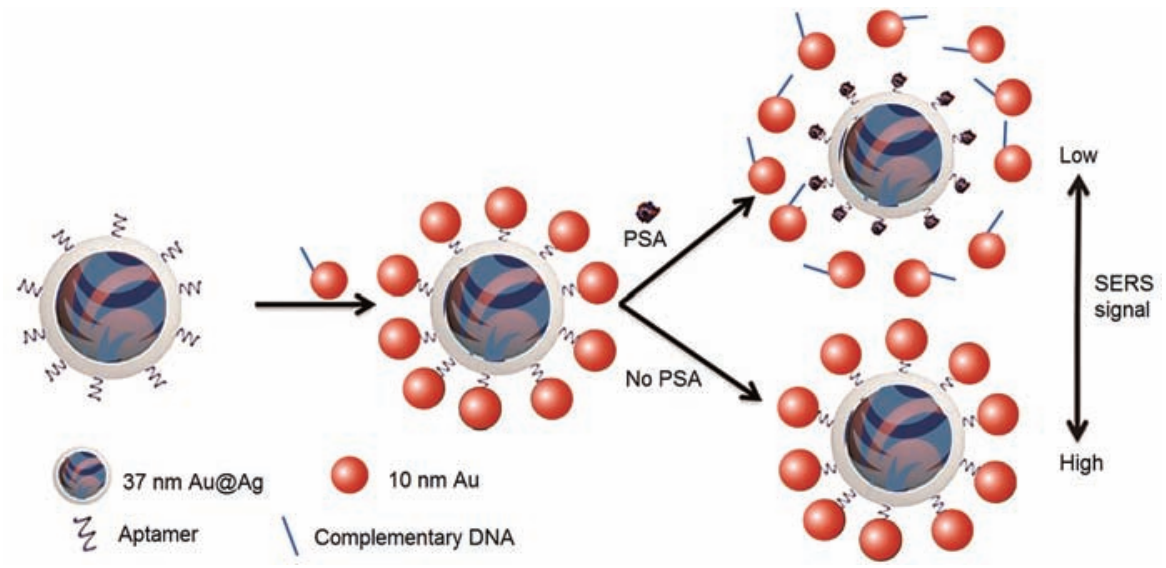

图 2 (网络版彩色)适配体介导纳米颗粒组装成核心-卫星结构 ${ }^{[21]}$

Figure 2 (Color online) Aptamar-mediated nanoparticles assemble to core-shell structures ${ }^{[21]}$

可以由SERS信号量化.

\section{2 抗原-抗体反应}

抗原-抗体反应是指抗原与相应抗体之间所发生
的特异性结合反应. 抗原-抗体之间具有高度专一的 选择性以及较强的识别性, 因此可以直接用来组装 等离子激元纳米颗粒.

Jiang 课题组 ${ }^{[22]}$ 利用抗原抗体识别作用介导 
AuNPs组装成网络结构 (图3). 先在AuNPs上联接活 性肽链, 然后在肽链上修饰组蛋白修饰酶, 加人特异 性免疫球蛋白 $\mathrm{IgG}$ 抗体后, 可以触发酶修饰的AuNPs 自组装形成网络结构, 从而使溶液产生直观的颜色 变化, 并且可以检测到等离子激元共振吸收峰的显 著变化. Kotov课题组 ${ }^{[23]}$ 用抗体功能化AuNPs, 利用 抗原-抗体免疫反应将AuNPs和银纳米颗粒组装成手 性异二聚体. $\mathrm{Xu}$ 等人 ${ }^{[24]}$ 首次通过免疫识别作用介导 纳米颗粒组装构建了通用的手性检测平台. 通过抗 原-抗体的免疫识别作用将 20 和 $10 \mathrm{~nm}$ 的AuNPs组装 成不对称的手性二聚体, 加人靶分析物双酚 $\mathrm{A}(\mathrm{BPA})$ 时, BPA与对应抗体竞争结合使AuNPs分散, 致使圆 二色性(CD)信号降低, 可实现对BPA的监测.

\section{3 生物素-亲和素系统}

生物素-亲合素系统(BAS)是一种新型生物反应 放大系统. 亲和素与生物素的结合反应呈现出高特 异性及高灵敏性, 使其在反应体系应用中用量极微, 并且生物素具有结合力强、体积小等优点, 因此BAS 也被广泛应用于介导组装等离子激元纳米颗粒.

Murphy课题组 ${ }^{[25,26]}$ 利用生物素与链霉亲和素的 特异性识别作用, 对AuNRs进行了一维、二维和三维 组装. Jun等人 ${ }^{[27]}$ 在大 AuNPs 上修饰亲和素, 在小 AuNPs上通过肽桥修饰生物素, 最后通过亲和素和 生物素特异性反应组装成卫星结构. Lee课题组 ${ }^{[28]}$ 也
利用类似的方法组装了卫星纳米颗粒组装体(图4). 首先, 生物素化的肽链通过硫醇介导联接到核心 AuNPs上, 此肽链含有丝氨酸胰蛋白酶活性位点; 随 后加人链需亲和素修饰的卫星纳米颗粒，生物素亲 和素特异性结合可诱导组装成卫星结构; 在丝氨酸 胰蛋白酶存在下, 肽链被打断, 卫星纳米颗粒与核心 纳米颗粒分离, 可通过暗场显微镜观察颗粒的散射 光谱图及颜色变化来监测该过程，从而监测丝氨酸 胰蛋白酶的活性.

\section{4 有机小分子和聚合物}

以有机小分子、嵌段共聚物、线性聚合物、树枝 状聚合物和超支化聚合物等作为交联剂, 利用有机 分子之间的静电作用、氢键、亲疏水作用等将等离子 激元纳米颗粒组装成二聚体、链状、球状、卫星状、 管状等特殊结构, 也是目前国内外研究的热点 ${ }^{[29,30]}$. 利用有机小分子和聚合物介导纳米颗粒组装可以对 组装体的结构、位置和尺度进行精确调控.

Yoon等人 ${ }^{[31]}$ 利用二硫醇作为联接剂(例如 1,10 癸二硫醇)组装了高纯度的不对称核心-卫星纳米组 装体. 先将 $51 \mathrm{~nm}$ 左右的核心AuNPs固定在玻璃片上, 再用烷烃二硫醇使AuNPs颈基功能化，随后将其浸 泡在13 nm的AuNPs溶液中组装成核心-卫星结构，最 后将此基片在乙醇中超声，核心-卫星结构会优先超 声下来形成不对称纳米组装体(图5). 此不对称纳米

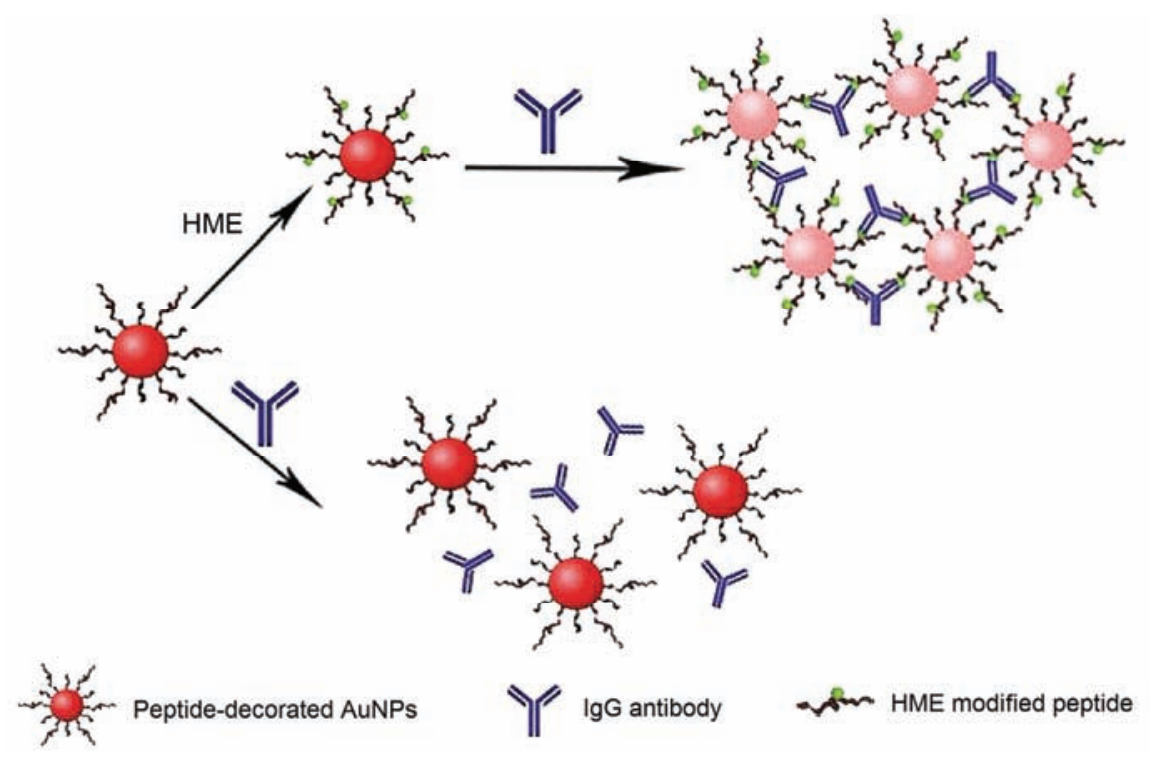

图 3 (网络版彩色)抗原-抗体反应介导的纳米颗粒组装 ${ }^{[22]}$

Figure 3 (Color online) Scheme of antigen-antibody mediated nanoparticles assemblies ${ }^{[22]}$ 


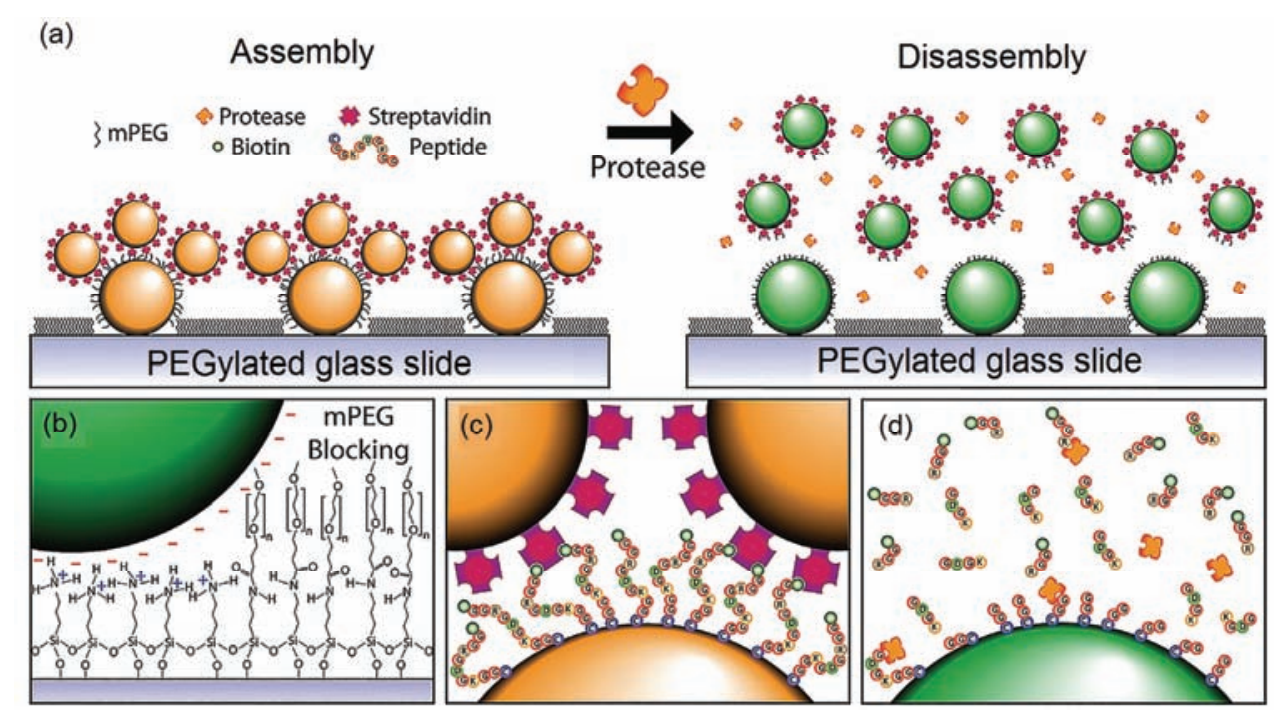

图 4 (网络版彩色)生物素-亲和素特异性结合介导AuNPs组装成功能性卫星结构. (a) 溶蛋白性裂解诱导AuNPs卫星结构组装体解离过程; (b) 带负电荷的AuNPs在玻璃表面固定后采用mPEG封闭活性位点; (c) 半胱胺酸/生物素-链霖亲合素体系联结卫星颗粒; (d) 胰蛋白酶裂解肽链释 放卫星颗粒过程用于比色法检测蛋白酶 ${ }^{[28]}$

Figure 4 (Color online) Biotin-avidin system mediated AuNPs functional assemblies. (a) Core-satellite Au nanoassemblies before and after proteolytic cleavage. (b) Immobilization of the negatively charged AuNP cores is enabled by Coulombic electrostatic attraction to glass surface. The glass slide is subsequently passivated with an mPEG chain to block nonspecific satellite adsorption. (c) Satellite attachment is enabled by a directional cysteine/biotin-streptavidin peptide tether. (d) Addition of trypsin cleaves the peptide, releasing the satellites into solution and enabling colorimetric detection of the protease ${ }^{[28]}$

结构具有强烈的表面等离子激元耦合效应, 并且耦 合强度与核心纳米颗粒和卫星纳米颗粒之间的距离 密切相关, 此不对称核心-卫星纳米组装体可以作为 一个很好的SERS增强基底. 后来, 该课题组将此组 装方法进行了扩展, 组装了异质金银核心-卫星纳米 组装体 ${ }^{[32]} 、 A u N P s$ 二聚体 ${ }^{[6]}$ 、金纳米立方体-金纳米 颗粒二聚体 ${ }^{[8]}$, 研究了核心纳米颗粒与卫星纳米颗粒 的间隙距离变化以及卫星纳米颗粒的数量等对等离 子耦合效应的影响.

此外, 线性聚合物, 如聚乙烯亚胺(PEI), 可以将 磺酸基团功能化的金纳米颗粒组装为立方或六边形 结构, 该结果也反证了 PEI存在 2 种晶体构型而导致 了金纳米颗粒的不同阵列形态 ${ }^{[33]}$. 当然, 用于调控 等离子激元组装应用最为广泛的还是嵌段聚合物, 如聚苯乙烯- $b$ - 聚甲基丙烯酸甲酯(PS- $b$-PMMA)、聚 苯乙烯- $b$ - 聚丙烯酸(PS- $b$-PAA)、聚苯乙烯- $b$ - 磷酸烯 醇式丙酮酸(PS- $b$-PEP) 等, 可有效地将金纳米颗粒自 组装为光谱可调的等离子激元薄膜 ${ }^{[34]}$. 通过自组装 方法, 树状聚合物分子也可以用于控制等离子激元 间距而调控共振耦合效应的媒介体. Rotello课题 组 ${ }^{[35]}$ 通过紫外光谱分析颗粒PAMAM样品, 证明了 组装大分子在调控颗粒间耦合而导致的极化现象的
应用前景.

\section{2 分子介导纳米颗粒组装体的生物应用}

相比于单独的纳米颗粒, 等离子激元纳米组装 体由于颗粒间的等离子激元耦合效应展现出许多新 颖或更强的性质; 并且纳米颗粒不同的组装方式、组 装体的纳米颗粒数目、组装纳米颗粒之间的距离大小 等会影响其等离子激元耦合的程度, 从而影响等离 子共振峰的位置和强度 ${ }^{[36,37]}$. 自组装等离子激元纳 米结构中相邻纳米颗粒之间热点的形成在生物传感、 生物成像、癌症治疗和医学诊断等方面都具有广泛的 应用前景 ${ }^{[38]}$.

\section{1 比色法生物传感}

AuNPs溶液的颜色与其分散状态密切相关, 当 AuNPs 由分散状态逐渐聚集时，由于颗粒间的表面 等离子共振偶合导致其颜色逐渐变化. 因此, 根据 AuNPs溶液外在颜色的变化可以构建比色法生物传 感器 ${ }^{[28,39]}$.

早在1997年Mirkin课题组 ${ }^{[40]}$ 就利用AuNPs 组装 前后溶液颜色的变化构建了对核酸的传感。在 AuNPs上修饰DNA, 当加人互补的靶DNA后, 由于 


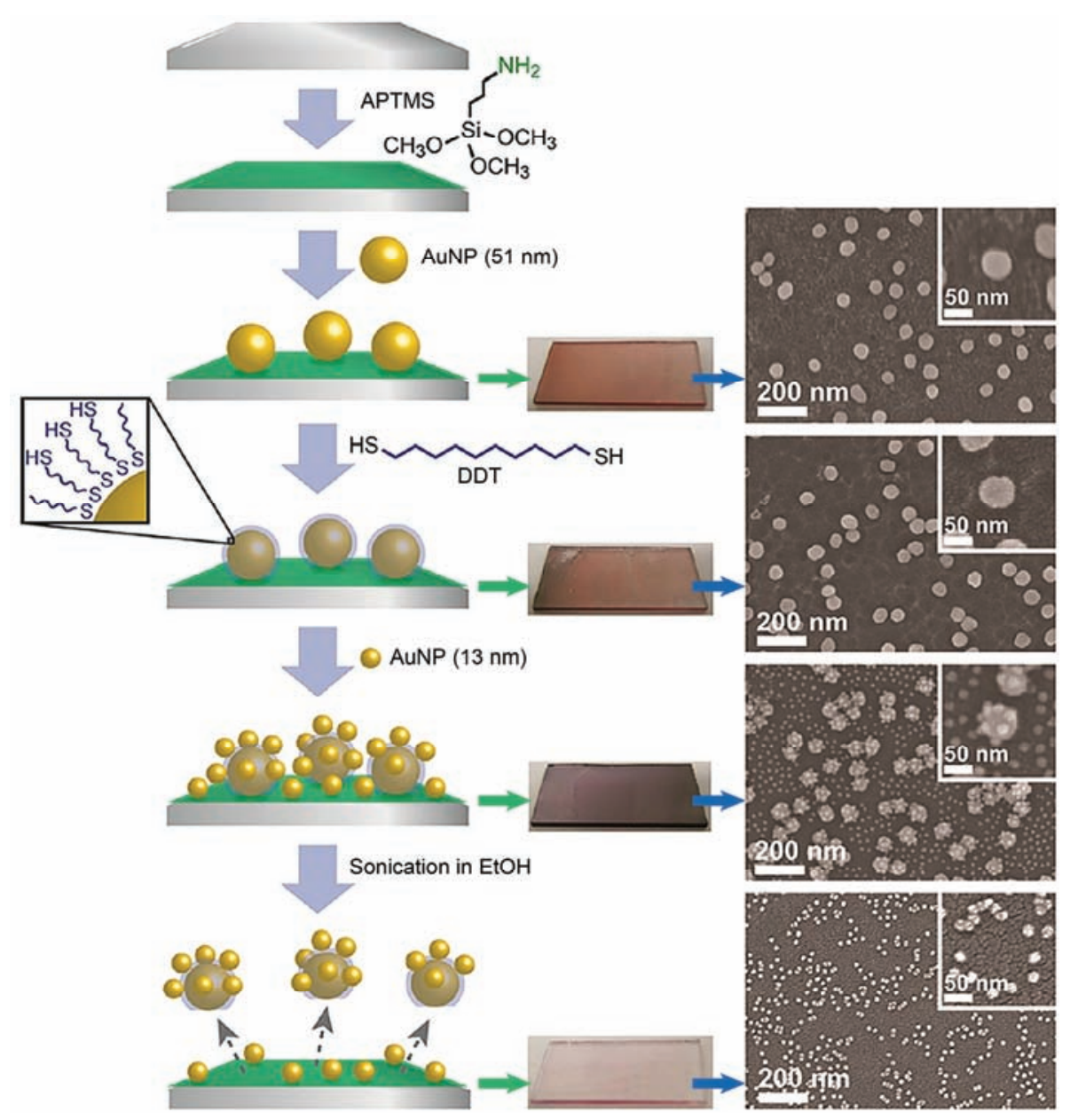

图 5 (网络版彩色)有机小分子介导的核-卫星结构纳米颗粒组装步骤 ${ }^{[31]}$

Figure 5 (Color online) Stepwise assembly process for asymmetric core-satellite nanoassemblies using selective desorption ${ }^{[31]}$

DNA杂交诱导AuNPs组装致使AuNPs溶液紫外吸收 峰发生红移, 溶液的颜色由红色变为蓝色, 从而实现 了对靶向DNA的检测. Park和Yeo ${ }^{[41]}$ 利用纳米等离子 激元卫星结构组装体建立了对 miR-21的比色传感. 用探针1修饰固定在玻璃片上的核心AuNPs, 再在溶 液中用探针 2 修饰卫星 AuNPs, 核心 AuNPs 与卫星 AuNPs 只有在靶miR-21存在时才会组装, 否则该组 装将无法进行, 使用暗场显微镜能观察到组装前后 颗粒散射颜色从绿色变为黄色, 通过颜色的变化可 以实现对 miR-21的检测(图6).

$\mathrm{Kim}$ 课题组 ${ }^{[42]}$ 通过设计 Y 型 DNA杂交形成 AuNPs二聚体, 构建了对靶DNA的比色传感, 检测限 比常用的苂光传感器低几个数量级, 比传统AuNPs 聚集传感器的灵敏度高 10000 倍. 基于此, Lin课题 组 ${ }^{[43]}$ 利用相反的过程构建了对微囊藻毒素 (MC) 的比

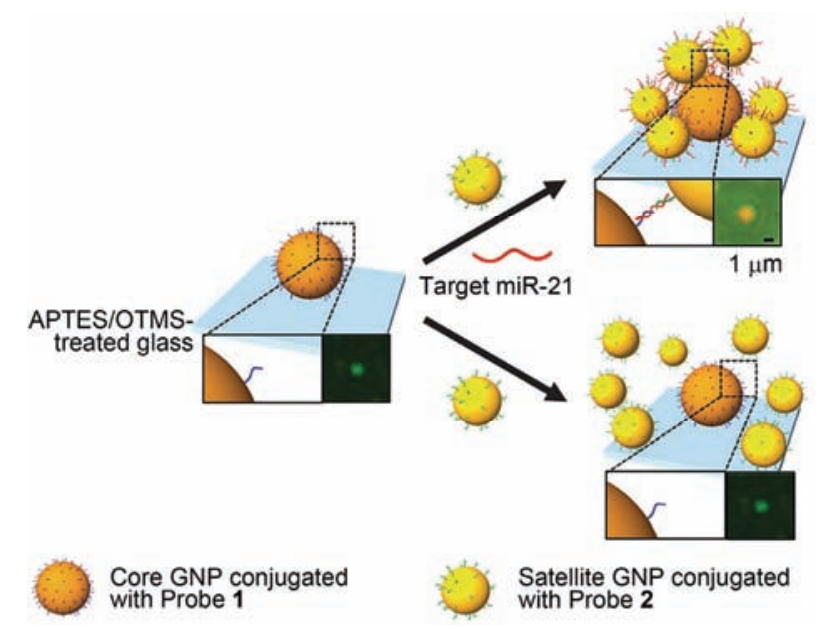

图 6 (网络版彩色)基于纳米等离子激元卫星结构对 $\mathrm{miR}-21$ 的比色 传感 ${ }^{[41]}$

Figure 6 (Color online) Colorimetric detection miR-21 based on plasmonic nanoparticles assemblies ${ }^{[41]}$ 
色传感, 与 $\mathrm{MC}$ 靶结合的适配体作为联接剂, 将 AuNPs组装成二聚体. 当MC存在时, $\mathrm{MC}$ 与适配体结 合致使AuNPs分离, 溶液的颜色由蓝变红, 从而实现 了对 $\mathrm{MC}$ 的比色传感, 检测限可达 $0.05 \mathrm{nmol} / \mathrm{L}$. 最近, Zhou 课题组 ${ }^{[44]}$ 精心设计了新颖的嵌段发夹探针 (DHPs), 结合高效的靶催化DHPs组装和AuNPs溶液 灵敏的比色分析实现了对靶DNA的超灵敏传感, 检 测限达 $0.1 \mathrm{pmol} / \mathrm{L}$, 可在 $0.01 \sim 5 \mathrm{pmol} / \mathrm{L}$ 范围内进行动 态检测.

\subsection{SERS生物传感}

SERS 检测技术由于其高灵敏度、高分辨率、不 破坏样品、可猝灭苂光、稳定性好及适合研究界面等 特点, 可广泛应用于环境、医药和生物检测等领域. 由于组装体中相邻两个纳米颗粒表面等离子激元耦 合效应, 导致颗粒间热点处的电磁场显著增强, 使位 于该处的拉曼信号获得数量级放大. 因此, 等离子激 元纳米颗粒组装体在 SERS 检测体系得到广泛应 用 ${ }^{[45 \sim 47]}$.

Tang课题组 ${ }^{[48]}$ 通过自组装方法制备了各种形貌 的二维和三维纳米组装结构, 组装结构的拉曼信号 增强因子最高可达 $10^{7}$, 比单独纳米颗粒的增强因子 高出几个数量级. Lin课题组 ${ }^{[49]}$ 将AuNPs 和 $\mathrm{Au}-\mathrm{Ag}$ 核 壳纳米粒子组装的卫星结构固定在硅片上, 可作为 很好的拉曼检测基底; 与单独AuNPs组装的卫星结 构基底相比, 此基底的拉曼增加强度高出 8 10倍. 因此, 利用组装结构作为拉曼检测基底, 可以大大提 高对分析物的检测灵敏度. Coronado课题组 ${ }^{[50]}$ 通过生 物素和亲和素相互作用构建了金纳米球(AuNSs)二聚 体, 二聚体产生的热点效应极大提高了对分析物的 检测灵敏度, 生物素的最低检测限能达到 $\mathrm{pmol} / \mathrm{L}$.

Kotov课题组 ${ }^{[51]}$ 用DNA对AuNRs进行选择性修 饰, 构建了不同构型的AuNPs和AuNRs组装体. 该组 装体与Hela细胞在 $37^{\circ} \mathrm{C}$ 下培养 $12 \mathrm{~h}$ 后, 发现纳米组装 体对细胞无毒, 并且检测到细胞培养液具有很强的 SERS信号, 这些信号峰来源于纳米组装体对脂类的 拉曼增强效应, 并且不同构型的组装体对脂类的拉 曼增强强度不同, 通过这些信号峰的强度可以对细 胞内的新陈代谢情况进行实时监测(图7).

\subsection{SPR生物传感}

在等离子激元纳米颗粒组装体中, 当颗粒间的
距离小于或等于光波长时所引起的近场耦合可使不 同的等离子激元产生相互作用 ${ }^{[9,52]}$. 组装体的形状、 尺寸、对称性以及颗粒间的间距和组成纳米颗粒的介 质决定了电荷极化的空间分布, 从而影响等离子激 元共振峰的分裂和相对位置 ${ }^{[53]}$. 利用分子介导等离 子激元纳米颗粒的组装可以根据组装前后等离子激 元共振峰的变化对生物分子进行传感. SPR生物传感 器具有灵敏度高、方便快捷、应用范围广、实时监控 等多项特点，已受到广泛关注.

Alivisatos课题组 ${ }^{[54]}$ 提出用“等离子标尺”研究单 链DNA杂交动力学. 金银纳米颗粒二聚体首先通过 单链DNA联接, 加人互补DNA链后, 观察到散射光 谱蓝移, 表明DNA链进行了杂交. 因为单链DNA相 对柔性, 引人互补DNA链进行杂交后使其刚性增强, 迫使纳米颗粒分离从而导致纳米颗粒二聚体的散射 光谱蓝移, 通过连续监测纳米颗粒二聚体的散射光 谱可以研究 DNA 的杂交动力学. Jun等人 ${ }^{[27]}$ 用含 DEVE序列的肽桥联接AuNPs组装成冠状结构, 在 caspase-3存在下, DEVE序列被打断致使AuNPs分离, 散射光谱发生蓝移, 因此通过散射光谱变化可以在 单分子水平检测 caspase-3. 实验结果表明，该等离子 激元标尺能够在 2 个多小时内连续监测 caspase- 3 的活 化, 为观察调亡细胞中 caspase-3 的早期活化提供了 足够的时间(图 8). Lee课题组 ${ }^{[5]}$ 通过生物分子特异性 反应(如抗原-抗体反应、生物素-亲合素系统)将星型 金纳米颗粒自组装成纳米星簇，提高了等离子分析 的灵敏性, 此结果在检测蛋白标志物的模型分析中 得到了验证. 他们发现减小亲和配体的尺寸对提高 分析灵敏性有很大的影响，此方法可方便地应用于 快速分子检测中.

\section{4 圆二色性生物传感}

手性纳米自组装结构是目前的研究热点之一. 手性纳米组装体在圆二色谱的可见光区会产生强烈 的手性信号, 并且此信号对组装体几何构型的变化 十分敏感, 即使非常微小的变化都会引起圆二色谱 的极大不同. 因此, 相比于其他传感器, 利用手性信 号作为检测信号操作简单、快速、无污染, 可用于生 物和医药等领域的超灵敏痕量检测 ${ }^{[56]}$.

许多等离子纳米颗粒组装体表现出等离子 $\mathrm{CD}$ 特 征，例如用氨基酸、单链DNA、DNA束和超分子纤维 支架等组装的AuNPs组装体. Kotov课题组 ${ }^{[57]}$ 通过聚 

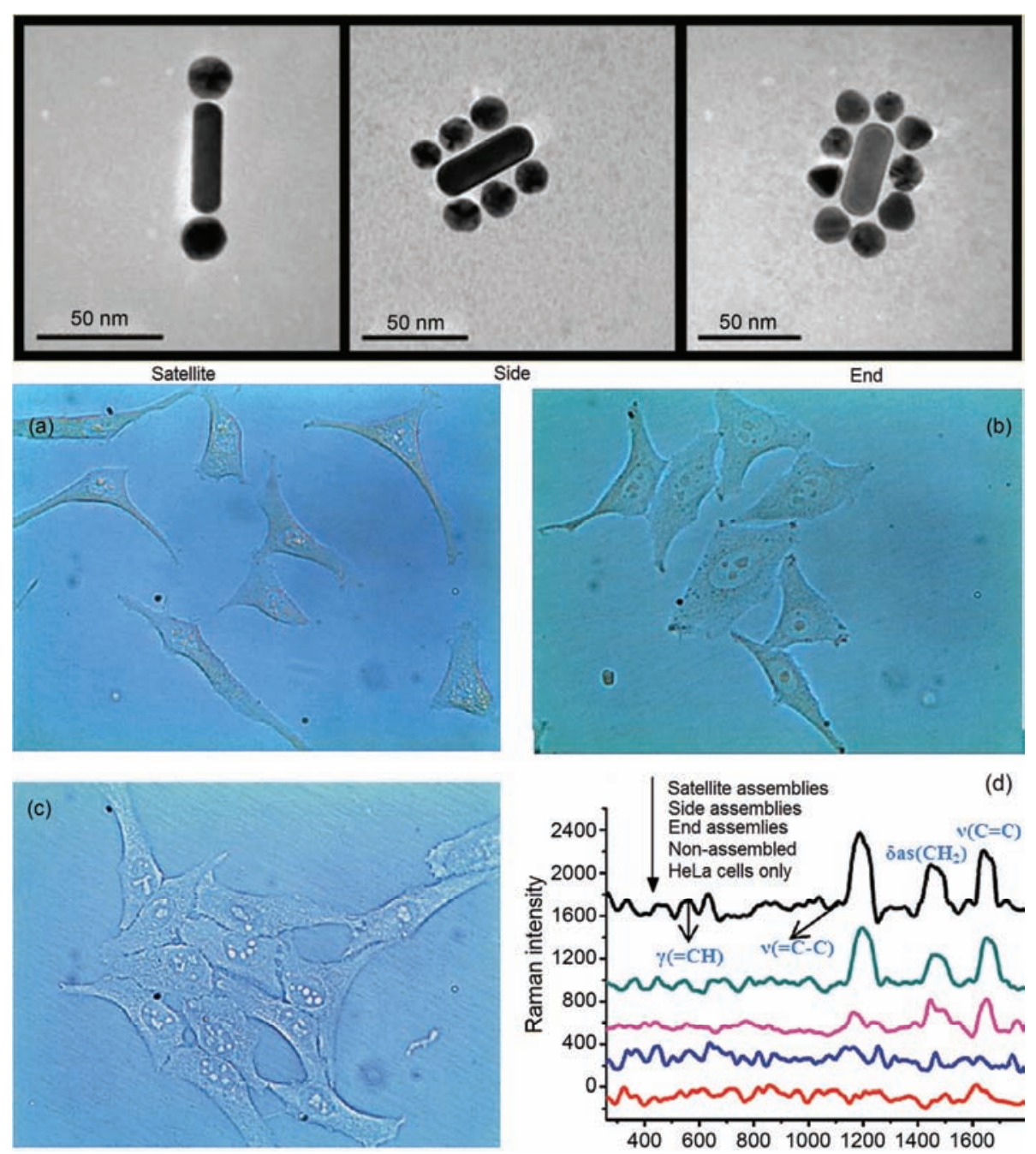

图 7 (网络版彩色)不同构型等离子组装体. 卫星结构(a), 侧边型(b), 两端型(c)在Hela细胞内的明场照片与相应的拉曼光谱(d) ${ }^{[51]}$

Figure 7 (Color online) Optical images of HeLa cell with different binary ensembles. Satellite (a), side (b), end (c) assemblies and Raman spectra (d) from the HeLa cells with three types of assemblies ${ }^{[51]}$

合酶链式反应(PCR)技术将AuNRs组装成具有手性信 号的肩并肩结构(图9), 基于此结构实现了对DNA的 超灵敏手性传感, 检测限可达 $3.7 \mathrm{amol} / \mathrm{L}$. 他们还利 用PSA的抗原抗体特异性识别作用将AuNPs和银纳 米颗粒组装成手性异二聚体, 建立了PSA的超灵敏 手性传感器 ${ }^{[23]}$. 随后, 他们先通过PCR技术将 25 和 10 $\mathrm{nm}$ 的AuNPs组装成手性二聚体, 然后在二聚体上沉 积金和银壳层，手性信号的放大结合 PCR技术的指 数放大，使核-壳组装体对 DNA 的检测限可达到 $\mathrm{zmol} / \mathrm{L}^{[58]}$.

\section{5 光热治疗及生物成像}

等离子激元纳米颗粒组装体可应用于治疗, 例
如光热癌症治疗. 贵金属纳米颗粒已被证明是非常 好的光热材料, 与单独的纳米颗粒相比，等离子激元 纳米颗粒组装体在激光照射后能产生更大、更强的热 能，热能再被传递到肿瘤组织，局部的、特定位点的 升温能杀伤肿瘤细胞, 从而可有效治疗癌症. Ji课题 组 ${ }^{[59]}$ 用带正电荷和带负电荷的放基配体折中的混合 电荷自组装单分子膜修饰AuNPs, 利用肿瘤组织酸 性 $\mathrm{pH}$ 条件诱导其聚集, 致使AuNPs聚集体的吸收峰 红移到近红外, 从而可用于光热治疗 (图 10). 最近, He课题组 ${ }^{[60]}$ 基于可生物降解的AuNRs 聚集体仿生胶 囊，有效结合了等离子纳米颗粒组装体的治疗应用 和生物成像能力, 开发出一个结合肿瘤成像和光热 治疗的新的治疗诊断平台 ${ }^{[61]}$. 
(a)
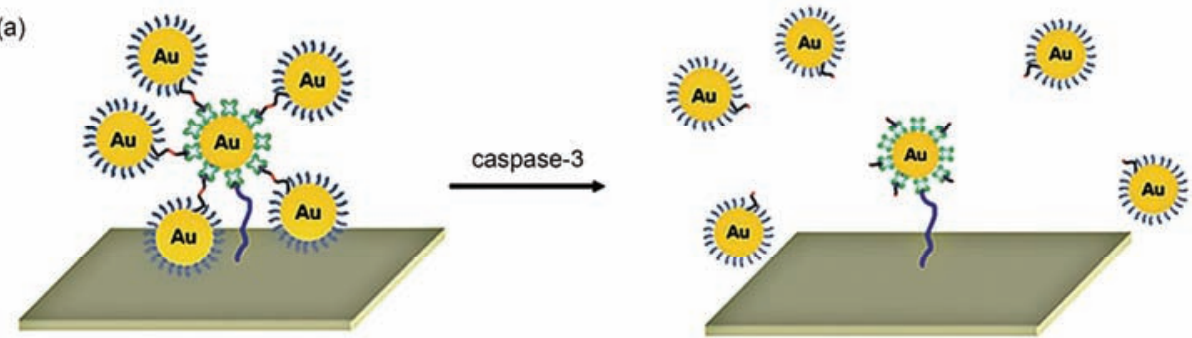

(b)

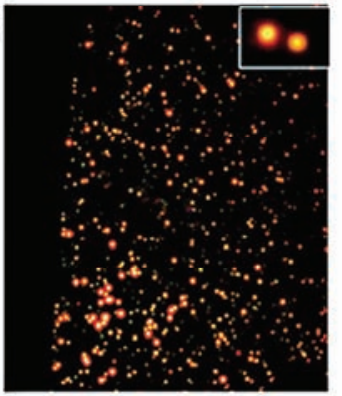

(c)

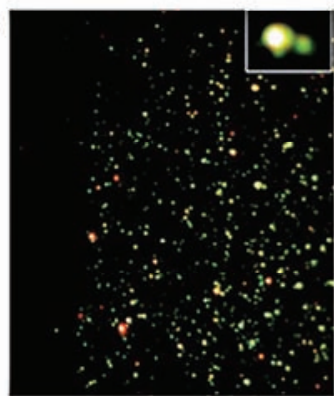

(d)

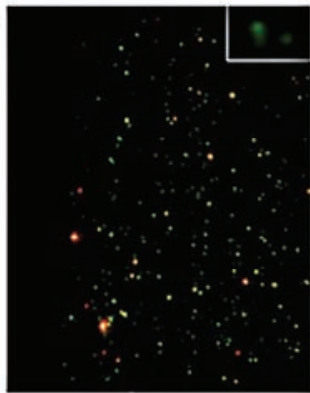

图 8 (网络版彩色)基于冠状纳米颗粒组装体在单分子水平连续检测caspase-3. (a) Caspase-3 切断肽链释放卫星颗粒的检测原理. 组装体(b)、中 间过程(c)和完全释放卫星颗粒后(d)的暗场光学照片 ${ }^{[27]}$

Figure 8 (Color online) In vitro caspase-3-mediated cleavage of crown nanoparticle plasmon rulers. (a) Schematic of experimental design. (b)-(d) After caspase- 3 molecules were introduced, and the color and intensity changes of crown nanoparticles were monitored under dark-field microscopy ${ }^{[27]}$

(a)

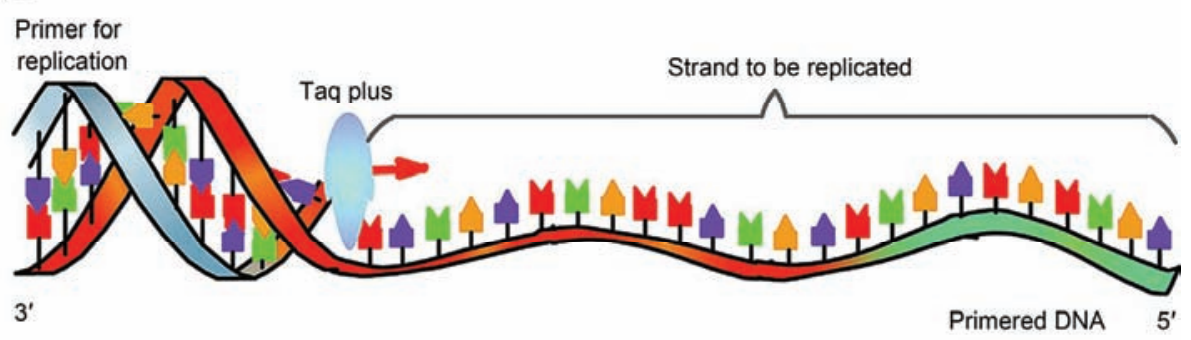

(b)
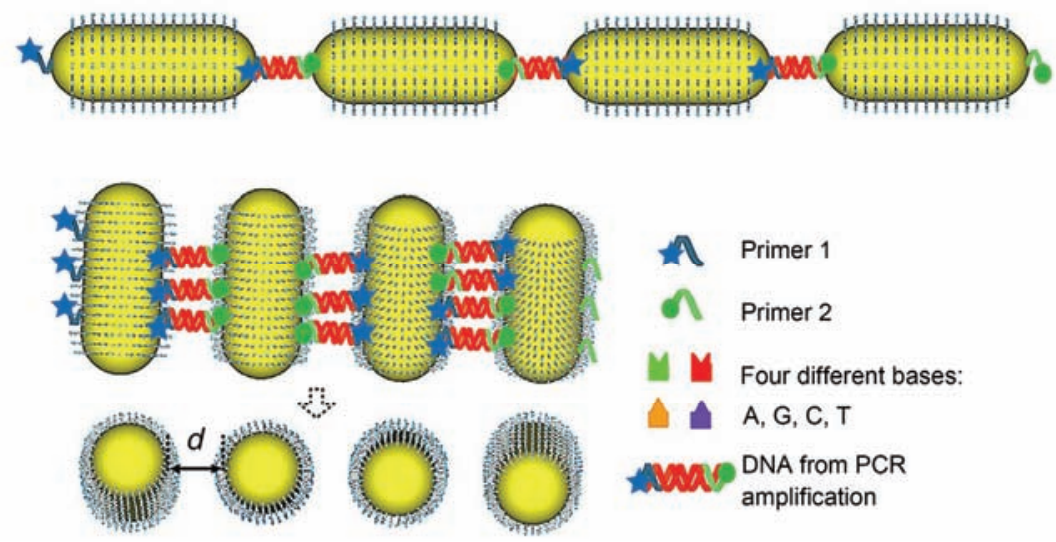

11 Four different bases:

$\Delta \mathrm{A}, \mathrm{G}, \mathrm{C}, \mathrm{T}$

What DNA from PCR
amplification

图 9 (网络版彩色)基于PCR技术的AuNRs自组装原理图. (a) PCR技术可复制DNA链通过引物、模板DNA、聚合酶和 4 种不同的DNA碱基. (b) 基于PCR技术的金纳米棒端对端组装. (c) 基于PCR技术的金纳米棒侧对侧组装, 其组装间隙为 $d^{[57]}$

Figure 9 (Color online) Scheme of AuNRs self-assemble methods based on PCR. (a) PCR replication procedure in which a DNA strand can be ampilified using primer, template DNA, taq plus polymerase and four different DNA bases. (b) PCR-based gold nanorods end-to-end assembly. (c) PCR-based gold NRs SBS assembly with inter-nanorod gap $d^{[57]}$ 


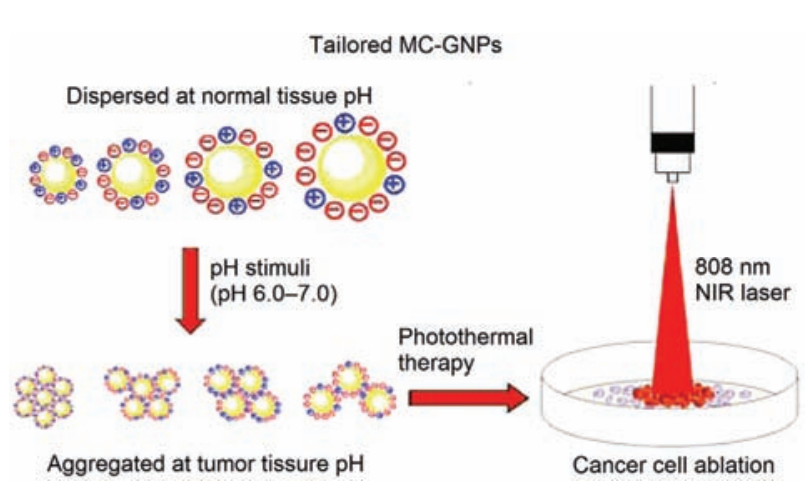

图 10 (网络版彩色)pH诱导AuNPs组装用于光热治疗 ${ }^{[59]}$

Figure 10 (Color online) $\mathrm{pH}$ induced photothermal therapy by AuNPs assembles $^{[59]}$

光声成像是近年来发展起来的一种新型成像技 术, 它很好地结合了光学成像和声学成像的优点, 是 一种非离子化、非侵入性的成像模式. 贵金属纳米颗 粒产生的光声信号来源于由光能转化的热能被转移 到周围环境而产生的压力瞬变. AuNPs自组装体能显 著增强光声信号, 这是因为相对于单个纳米颗粒, 组 装体能提高热传递速率, 增强的光声信号可为活体 光声成像技术提供更高的分辨率 ${ }^{[62]}$.

拉曼成像技术是通过采集一定样品区域中的拉 曼信号来获得样品详细化学图像的一种探测方法. 拉 曼成像能在可见光至近红外光谱区域工作, 能够有效 避免自发荧光干扰, 这些优势使其在活细胞成像中有 更大的应用潜力 ${ }^{[63]}$. 贵金属纳米颗粒是良好的 SERS 基底, 组装体中由于相邻 2 个纳米颗粒表面等离子激 元耦合效应导致颗粒间热点处的电磁场显著增强, 并 且热点增加, 耦合效应增强, 因此AuNPs组装体能更 好地用于拉曼成像, 并实现单分子水平的检测 ${ }^{[64]}$.

\section{3 总结与展望}

自组装技术可以将单个纳米颗粒组装成一维、二 维或三维空间构象的新型复合材料, 基于颗粒间的 等离子共振耦合效应, 会展现出许多新颖或更强的 物理、光学性质, 因此, 纳米颗粒自组装技术在纳米 科学领域越来越受到关注. 利用分子介导等离子激 元纳米颗粒组装成具有固定结构的组装体是目前研 究最多的组装方式, 可用于介导等离子激元纳米颗 粒组装的主要有DNA、抗原-抗体反应、生物素-亲和 素系统、有机小分子和聚合物等.

目前, 关于分子介导组装多形貌纳米结构的报 道层出不穷, 但是分子介导纳米颗粒的组装仍存在 一些有待突破的难题. 主要面临以下几个方面的挑 战：(1) 通过设计介导分子的结构以及调节分子间的 作用力, 获得预想可控的纳米材料超结构, 并且使这 种超结构具有可控的手性及良好的实用性仍然是需 要突破的难点; (2) 高产率地制备确定尺寸、几何形 状以及化学异质性的等离子激元纳米颗粒组装体的 方法仍需进一步的研究; (3) 实时监控组装过程的表 征技术及对组装过程热力学和动力学的理论分析, 为组装方法提供指导和设计准则 ${ }^{[11,38]}$.

分子介导的自组装技术可以很好地控制等离子 激元纳米颗粒的空间排布及组装体的结构外形，使 其在生物传感、成像和治疗等方面有着重要的应用价 值. 我们相信随着纳米颗粒自组装方法的持续发展, 基于金属纳米颗粒组装体表面等离子激元理论框架 的深人研究, 等离子激元纳米颗粒组装体将在化学、 物理、光学以及生物医学等领域发挥重要的研究意义 与应用价值.

\section{参考文献}

1 Ritchie R. Plasma losses by fast electrons in thin films. Phys Rev, 1957, 106: 874

2 Xi C, Marina P F, Xia H, et al. Directed self-assembly of gold nanoparticles into plasmonic chains. Soft Matter, 2015, 11: 4562-4571

3 Li K, Wang K, Qin W, et al. DNA-directed assembly of gold nanohalo for quantitative plasmonic imaging of single-particle catalysis. J Am Chem Soc, 2015, 137: 4292-4295

4 Sannomiya T, Hafner C, Voros J. In situ sensing of single binding events by localized surface plasmon resonance. Nano Lett, 2008, 8: $3450-3455$

5 Toma S H, Santos J J, Araki K, et al. Pushing the surface-enhanced Raman scattering analyses sensitivity by magnetic concentration: A simple non core-shell approach. Anal Chim Acta, 2015, 855: 70-75

6 Cha H, Yoon J H, Yoon S. Probing quantum plasmon coupling using gold nanoparticle dimers with tunable interparticle distances down to the subnanometer range. ACS Nano, 2014, 8: 8554-8563

7 Akiyama Y, Shikagawa H, Kanayama N, et al. Modulation of interparticle distance in discrete gold nanoparticle dimers and trimers by 
DNA single-base pairing. Small, 2015, 11: 3153-3161

8 Lee D, Yoon S. Gold nanocube-nanosphere dimers: Preparation, plasmon coupling, and surface-enhanced Raman scattering. J Phys Chem C, 2015, 119: 7873-7882

9 Klinkova A, Choueiri R M, Kumacheva E. Self-assembled plasmonic nanostructures. Chem Soc Rev, 2014, 43: 3976-3991

10 Thorkelsson K, Bai P, Xu T. Self-assembly and applications of anisotropic nanomaterials: A review. Nano Today, 2015, 10: 48-66

11 Ma W, Xu L, Wang L, et al. Orientational nanoparticle assemblies and biosensors. Biosens Bioelectron, 2016, 79: 220-236

12 Chao J, Lin Y, Liu H, et al. DNA-based plasmonic nanostructures. Mater Today, 2015, 18: 326-335

13 Mirkin C A, Letsinger R L, Mucic R C, et al. A DNA-based method for rationally assembling nanoparticles into macroscopic materials Nature, 1996, 382: 607-609

14 Alivisatos A P, Johnsson K P, Peng X, et al. Organization of "nanocrystal moleculesusing” DNA. Nature, 1996, 382: 609-611

15 Park S Y, Lytton-Jean A K, Lee B, et al. DNA-programmable nanoparticle crystallization. Nature, 2008, 451: 553-556

16 Schreiber R, Do J, Roller E M, et al. Hierarchical assembly of metal nanoparticles, quantum dots and organic dyes using DNA origami scaffolds. Nat Nanotechnol, 2014, 9: 74-78

17 Zheng Y, Li Y, Deng Z. Silver nanoparticle-DNA bionanoconjugates bearing a discrete number of DNA ligands. Chem Commun, 2012, 48: 6160-6162

18 Yoon B, Luedtke W, Barnett R N, et al. Hydrogen-bonded structure and mechanical chiral response of a silver nanoparticle superlattice. Nat Mater, 2014, 13: 807-811

19 Pei H, Li F, Wan Y, et al. Designed diblock oligonucleotide for the synthesis of spatially isolated and highly hybridizable functionalization of DNA-gold nanoparticle nanoconjugates. J Am Chem Soc, 2012, 134: 11876-11879

20 Yao G, Pei H, Li J, et al. Clicking DNA to gold nanoparticles: Poly-adenine-mediated formation of monovalent DNA-gold nanoparticle conjugates with nearly quantitative yield. NPG Asia Mater, 2015, 7: e159

21 Ma W, Yin H, Xu L, et al. Ultrasensitive aptamer-based SERS detection of PSAs by heterogeneous satellite nanoassemblies. Chem Commun, 2014, 50: 9737-9740

22 Zhen Z, Tang L J, Long H, et al. Enzymatic immuno-assembly of gold nanoparticles for visualized activity screening of histone-modifying enzymes. Anal Chem, 2012, 84: 3614-3620

23 Wu X, Xu L, Liu L, et al. Unexpected chirality of nanoparticle dimers and ultrasensitive chiroplasmonic bioanalysis. J Am Chem Soc, 2013, 135: 18629-18636

$24 \mathrm{Xu} \mathrm{Z,} \mathrm{Xu} \mathrm{L,} \mathrm{Zhu} \mathrm{Y,} \mathrm{et} \mathrm{al.} \mathrm{Chirality} \mathrm{based} \mathrm{sensor} \mathrm{for} \mathrm{bisphenol} \mathrm{A} \mathrm{detection.} \mathrm{Chem} \mathrm{Commun,} \mathrm{2012,} \mathrm{48:} \mathrm{5760-5762}$

25 Caswell K, Wilson J N, Bunz U H, et al. Preferential end-to-end assembly of gold nanorods by biotin-streptavidin connectors. J Am Chem Soc, 2003, 125: 13914-13915

26 Gole A, Murphy C J. Biotin-streptavidin-induced aggregation of gold nanorods: Tuning rod-rod orientation. Langmuir, 2005, 21: 10756-10762

27 Jun Y W, Sheikholeslami S, Hostetter D R, et al. Continuous imaging of plasmon rulers in live cells reveals early-stage caspase-3 activation at the single-molecule level. Proc Natl Acad Sci USA, 2009, 106: 17735-17740

28 Waldeisen J R, Wang T, Ross B M, et al. Disassembly of a core-satellite nanoassembled substrate for colorimetric biomolecular detection. ACS Nano, 2011, 5: 5383-5389

29 Das S, Ranjan P, Maiti P S, et al. Dual-responsive nanoparticles and their self-assembly. Adv Mater, 2013, 25: 422-426

30 Xiong L, Shuhendler A J, Rao J. Self-luminescing BRET-FRET near-infrared dots for in vivo lymph-node mapping and tumour imaging. Nat Commun, 2012, 3: 1193

31 Yoon J H, Lim J, Yoon S. Controlled assembly and plasmonic properties of asymmetric core-satellite nanoassemblies. ACS Nano, 2012, 6: 7199-7208

32 Yoon J H, Zhou Y, Blaber M G, et al. Surface plasmon coupling of compositionally heterogeneous core-satellite nanoassemblies. J Phys Chem Lett, 2013, 4: 1371-1378

33 Schmid G, Bäumle M, Beyer N. Ordered two-dimensional monolayers of Au55 clusters. Angew Chem Int Ed, 2000, 39: 181-183

34 Ofir Y, Samanta B, Rotello V M. Polymer and biopolymer mediated self-assembly of gold nanoparticles. Chem Soc Rev, 2008, 37: $1814-1825$

35 Srivastava S, Frankamp B L, Rotello V M. Controlled plasmon resonance of gold nanoparticles self-assembled with PAMAM dendrimers. Chem Mater, 2005, 17: 487-490

36 Funston A M, Novo C, Davis T J, et al. Plasmon coupling of gold nanorods at short distances and in different geometries. Nano Lett, 2009, 9: 1651-1658

37 Jain P K, Eustis S, El-Sayed M A. Plasmon coupling in nanorod assemblies: Optical absorption, discrete dipole approximation simulation, 
and exciton-coupling model. J Phys Chem B, 2006, 110: 18243-18253

64 Shiohara A, Wang Y, Liz-Marzan L M. Recent approaches toward creation of hot spots for SERS detection. J Photochem Photobiol C, $2014,21: 2-25$ 


\title{
Plasmons nanoparticles assembly and biological applications
}

\author{
ZHANG Lei, MO Lan, SHEN JingJing, ZHANG Ying \& WANG LianHui \\ The Synergetic Innovation Center for Advanced Materials, Key Laboratory for Organic Electonics and Information Displays, Institute of Advanced \\ Matrials (IAM), Nanjing University of Posts \& Telecommunications, Nanjing 210023, China
}

Surface plasmon resonance (SPR) effect of nanoparticles, is an emerging subfield of nanophontonics, and it attracts increasing attention because of its potential applications in controlling and manipulating light at nanoscale dimensions. This review focuses on the molecules mediated nanoparticle assemblies and their biological applications, highlights several molecules mediated nanoparticle assembly technique and their recent applications in biomedical, and prospects the future development. Based on the specific binding ability of these functional mediate molecules (including DNA, antigen-antibody, aptamar, biotin-avidin, small organic molecules and polymers), plasmonic nanoparticles could be synthesized by self-assemble methods. Noble metal nanoparticle assemblies have broad applications in biological sensing, imaging and treatment due to their exclusive plasmon optical properties. With the rapid development of self-assembly technology, molecule-mediated self-assembly technology can be used well to control the spatial arrangement structure of plasmonic nanoparticles. Individual nanoparticle has been used to controllable assembled into one-dimensional, twodimensional or three-dimensional conformation of the novel composite materials. SPR effect of noble metal nanoparticles, is an emerging subfield of nanophontonics, and it attracts increasing attention because of its potential applications in controlling and manipulating light at nanoscale dimensions. Compared with the individual nanoparticle, nanoparticle assemblies have a lot of novel or excellent physical properties which exhibite convenient adjustable ability in SPR spectra due to noble metal nanoparticles with irregular shape. They show notable antenna effect at the edge of nanoparticles and the coupling effect between adjacent two nanoparticles. All of these unique optical properties on plasmonic nanoparticles will be further enhanced by the self-assemble nanotechnology, which exhibits many promising applications in the field of biosensors (including photocolorimetric method, SPR, surface-enhanced Raman scattering (SERS), circular dichroism etc.), photoacoustic imaging and photothermal therapy in recently decades. This review focuses on the molecules mediated nanoparticle assemblies and their biological applications, highlights several molecules mediated nanoparticle assembly technique and their recent applications in biomedical, and prospects the future development. Based on the specific binding ability of these functional mediate molecules (including DNA, antigenantibody, aptamar, biotin-avidin, small organic molecules and polymers etc.) plasmonic nanoparticles clusters could be synthesized to molecules mediated nanoparticle assemblies (MMNAs) by self-assemble methods following the well-designed linker. MMNAs could be widely used in bionanotechnology and biomedicine. The MMNAs would exhibit more sensitivity to the imperceptible changes of micro-environment around the assembles. There would be larger shifts in this resonance due to changes in the local index of refraction upon adsorption to the nanoparticles which could be used to detect biopolymers such as DNA or proteins. For example, the satellite gold nanoparticles linked on the surface of core particle by DEVD peptide would be cleaned by caspase- 3 from the core. This method has been used directly for real-time monitoring the enzymatic reaction by MMNAs' scattering spectra shifts. In addition, there would be abundant hot-spots between the satellite nanoparticles and the core, which is emerging as a powerful tool for SERS spectroscopic detection of trace molecular, cellular, and in vivo targets. The enhancement factors could achieve up to 1014-1015 by properly adjusting the LSPR spectroscopic profile and structural features of the MMNAs. What's interesting is that when noble metal nanoparticles aggregate, there has been a red-shift in the light absorption spectrum, which dramatically enhances the light absorbance in the NIR region. And the MMNAs would show great adjustable ability in plasmon resonance absorption which opening up new opportunities for plasmonic nanoparticles to be utilized as photosensitizers in the NIR photothermal therapy photoacoustic imaging applications. Based on the further comprehensive researches and developments of MMNAs in recent decades, we believe that the continued development of MMNAs will be applied well in the fields of chemistry, physics, optics and biomedicine in the future.

nanoparticles, self-assembly, molecules mediated, biological applications 